\title{
NACHFRAGEN IM DEUTSCHEN UND IM POLNISCHEN AM BEISPIEL LITERARISCHER DIALOGE UND IHRER ÜBERSETZUNGEN
}

ABSTRACT: Echo questions in German and Polish on the basis of literary dialogues and their translation

The paper deals with two types of German and Polish echo-questions (so called Rückfragen and Gegenfragen) and their communicative functions. It will be discussed which linguistic means can be used to achieve these functions in both languages.

KEYWORDS: question-answer-sequence; echo-question; communicative function

0.

„In zwischenmenschlicher Kommunikation kommt es, abgesehen vom Monolog in einer Interaktion, in jeder Sprache zum Rollenwechsel zwischen dem jeweiligen Sprecher und wenigstens einem seiner Hörer/Hörerinnen. Der Sprecherwechsel wird also als ein allgemein kommunikatives Prinzip betrachtet, das zur Gliederung des Textes [...] verwendbar ist" (Darski 22004: 25).

Solche durch den Sprecherwechsel bestimmte Sequenzen sind meist zweigliedrig und werden in der Konversationsanalyse als Paarsequenzen bezeichnet (vgl. Meibauer 22001: 134), unter denen Frage-Antwort-Sequenzen prototypisch sind. Diese können problemlos verlaufen oder von einer ähnlichen Zwischen- bzw. Zusatzsequenz unterbrochen sein, die aus einer Nachfrage und Antwort auf sie besteht. In der neueren Literatur werden Nachfragen allgemein definiert „als Fragen, die sich auf vorausgehende Äußerungen der Gesprächspartner beziehen" (Rost-Roth 2003: 326). Demnach sind Nachfragen keine autonomen, sondern reaktive Fragehandlungen, die auf Mitteilungen oder Aufforderungen als Voräußerungen folgen oder auf Voräußerungen, die selbst Fragen, d. h. Entscheidungs- oder Ergänzungsfragen sind. In der grammatischen Terminologie von Engel sind die von Mitteilungen oder Aufforde-

Czesława Schatte - Uniwersytet im. Adama Mickiewicza, Poznań, czescha@amu.edu.pl

Christoph Schatte - Uniwersytet im. Adama Mickiewicza, Poznań, cees@amu.edu.pl 
rungen induzierten Fragen als „Rückfragen“ bezeichnet, die auf Fragen folgenden als „Gegenfragen“ (vgl. Engel 1988: 55ff.; Engel ${ }^{42009: ~ 229 f f . ; ~ E n g e l ~ u . ~ a . ~ 1999: ~ 45, ~}$ Schatte/Schatte 2005).

\section{1.}

In linguistischen Arbeiten werden Nachfragen recht verschieden bezeichnet, so $u$. a. als Vergewisserungs- bzw. Versicherungsfragen, Nach- oder Gegenfragen, Reparaturfragen, Echofragen, assertive Fragen, Rückfragen im engeren und im weiteren Sinne (vgl. u. a. Maibauer 1987a, 1987b; Wunderlich 1986, Altmann 1987, Selting 1987, 1995; Engel 1988, ${ }^{4}$ 2009, Engel u. a. 1999, Reis 1992, Peretti 1993). Am üblichsten scheinen die Bezeichnungen „Rückfrage“ und „Echofrage“ zu sein. Die aufgezählten Bezeichnungen sind jedoch nicht synonym, weil sich die jeweiligen Fragen zum Teil im Typ der Voräußerung und in ihrer Struktur selbst unterscheiden. Meist legt die der jeweiligen Untersuchung zugrunde liegende Definition erst fest, welchen Bezugsbereich die Bezeichnungen haben.

Untersuchungen zu Nachfragen, ihren Formen und kommunikativen Funktionen sind nicht zahlreich. Die meisten von ihnen sind im Rahmen pragmatischer Analysen gesprochener Sprache, der Dialogforschung und Konversationsanalyse entstanden (vgl. Literaturverweise oben). In Grammatiken des Deutschen und des Polnischen werden sie generell kurz unter den Fragearten behandelt (vgl. Engel 1988, Engel ${ }^{4} 2009$, Engel u. a. 1999, Zifonun/Hoffmann/Strecker 1997, Helbig/Buscha ${ }^{15}$ 2001, Nagórko 2010). Eine umfassende Analyse reaktiver Frageakte des Deutschen legte neulich Rost-Roth vor (vgl. Rost-Roth 2000, 2003, 2011). In ihrer kritischen Diskussion der wichtigsten bisherigen Darstellungen zu Nachfragen geht die Autorin auf Probleme der Terminologie, der Struktur unter besonderer Berücksichtigung der Stellung des Finitums (falls vorhanden) und der Typologie ein und illustriert ihre Ausführungen mit übersichtlichen tabellarischen Zusammenstellungen (vgl. Rost-Roth 2003, Kap. 2, 3, 4; auch Rost-Roth 2011). Der akribische Vergleich analysierter Arbeiten erlaubt es, folgende Schlussfolgerungen zu ziehen:

Ein Grund dafür, dass sich Widersprüche und Unstimmigkeiten zeigen, ist darin zu sehen, dass nicht immer deutlich zwischen Form und Funktion unterschieden wird und zudem vielfach mit Intuitionen gearbeitet wird. Viele Nachfragen sind ohne Kontext jedoch mit unterschiedlichen Intonationen und dadurch auch unterschiedlichen Funktionen vorstellbar. Zudem sind Kontexte - [...] - schwer rekonstruierbar. (Rost-Roth 2011: 95)

Die frühere kontrastiv angelegte Arbeit von Peretti rechnet Nachfragen (dort „Rückfragen“ genannt) zu „,fremdinitiierten Reparaturen“, mit denen „ein Interaktionsteilnehmer auf ein Verständigungsproblem in einer Partneräußerung aufmerksam [macht] “ (Peretti 1993: 50), womit bereits die Definition auf die Grundfunktion solcher Fragen 
verweist. Vereinfachend und unter Berücksichtigung der von Engel (1988: 55; ${ }^{42009}$ : 231f.) eingebrachten Unterscheidung lässt sich die dialogische Verankerung von Rückund Gegenfragen wie folgt darstellen:

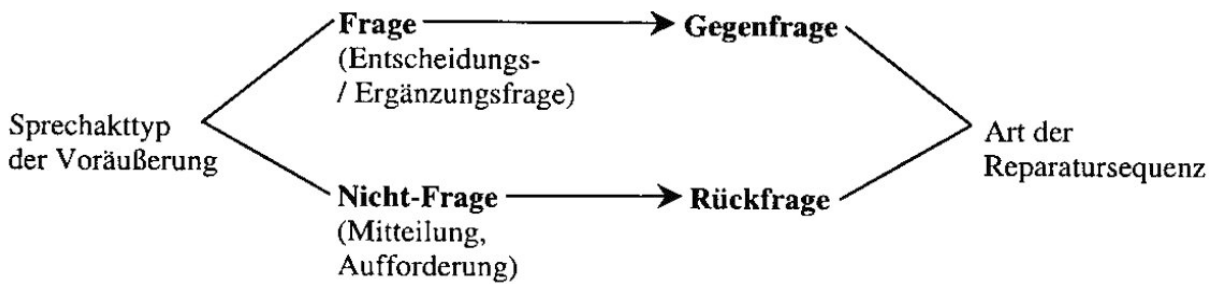

Der nachfolgende Dialog illustriert die Reaktionen mit einer Rückfrage auf eine Mitteilung als auch mit einer Gegenfrage auf eine Frage.

A: Daß Frauen aber auch immer alles gleich so übertreiben müssen!

B: Frauen?

A: Manche Frauen?

B: Welche Frauen?!

A: Du, zum Beispiel - Er spritzte.

B: Ich gebe mich nur nicht gerne mit halben Sachen zufrieden.

A: Nein? Spritz.

B: Nein! Spritz. (SW 250)

A: Że też kobiety wciąż muszą tak przesadzać!

B: Kobiety?

A: Niektóre kobiety?

B: Które kobiety?

A: Ty, na przykład - ochlapał mnie jeszcze raz.

B: Nie lubię zatrzymywać się w połowie drogi.

A: Nie? - znów krople wody.

B: Nie! - znów krople wody. (SB 295)

Impuls für die Äußerung einer Reparatursequenz in Form einer Rückfrage oder Gegenfrage ist also eine Voräußerung des Dialogpartners, die der Rezipient entweder hinsichtlich ihres Inhalts, ihrer Intention oder äußerer Gegebenheiten nicht (völlig) verstanden hat bzw. mit seinem Frageakt anzweifeln oder sogar zurückweisen will. Dabei wird zunächst von anderen Gründen abgesehen, wie z. B. wenn der Gesprächspartner eine Rück- oder Gegenfrage evtl. auch deshalb äußert, weil er Zeit gewinnen möchte, um seinen eigentlichen reaktiven Sprechakt vorzubereiten.

Rost-Roth unterscheidet in ihrer Untersuchung insgesamt vier Funktionen der Nachfragen, die auch in den meisten bisherigen Arbeiten - unabhängig von der Terminologie und der weiteren Gliederung in Rück- und Gegenfrage - be- bzw. angesprochen wurden: „Signalisierung von Verständnisproblemen, Signalisierung von 
Erwartungsproblemen, Vergewisserungsfunktionen und weitere Nachfragefunktionen“ (Rost-Roth 2003: 352).

Auf diese Funktionen soll im Folgenden im Kontext literarischer Dialoge und ihrer Übersetzungen ins Polnische eingegangen werden, um festzustellen, ob und wie die einzelnen Funktionen in der anderen Sprache realisiert werden und welchen Mehrwert sie in literarische Texte einbringen können. Alle Belege stammen aus einem dialogreichen deutschen Roman (vgl. Quellen), in dem Situations- und Sprachhumor eine bedeutende Rolle spielen. In den angeführten Belegen sind die Dialogpartner in den einzelnen Sequenzen mit Buchstaben A und B gekennzeichnet und die den Dialog begleitenden Situationsbeschreibungen berücksichtigt. In eckigen Klammern sind Auslassungen im Text markiert [...], kurze Angaben zur Situation [Telefongespräch] sowie kursiv die in der Übersetzung ausgelassenen Textstellen [fehlender Text] hinzugefügt. In runden Klammern stehen die Seitenangaben mit dem Sigle des Originals (SW) und der Übersetzung (SB). Rück- und Gegenfragen sowie Antworten auf diese sind fett gekennzeichnet, die übrigen Druckkennzeichnungen wie Kapitälchen entsprechen dem Original. Nicht eingegangen wird auf Differenzen zwischen Original und Translat, wenn sie für das Problem der Nachfrage nicht relevant sind, zumal es sich um eine literarische Übersetzung mit ästhetischem Mehrwert handelt. Da im Mittelpunkt der Untersuchung die bereits erwähnten Funktionen der Nachfragen stehen, werden ihre typischen sprachlichen Formen im Deutschen und im Polnischen in der einleitenden allgemeinen Charakteristik der jeweiligen Funktion vergleichend gegenübergestellt.

\section{2.}

Die grundlegende kommunikative Funktion der Nachfragen besteht nach Kallmeyer (1977: 52) in der „Sicherung der Verständlichkeit“ einer Voräußerung, von Rost-Roth (2003: 352) als „Signalisierung von Verständnisproblemen“ verstanden. Sie „signalisieren u. a. das Misslingen einer vorausgegangenen Äußerung und bezwecken die Herstellung bzw. Sicherung von Voraussetzungen, die für das Verstehen und Akzeptieren bzw. Ablehnen der betreffenden Äußerung notwendig sind“" (Peretti 1993: 48).

Nachfragen verlangen - wie jede Frage - nach einer Antwort, mit der das Verständnisproblem geklärt und ihre Rolle im Gespräch beendet ist, so dass das Gespräch an der Stelle fortgesetzt werden kann, an der es durch die Nachfrage unterbrochen wurde. Die Sequenz aus der Nachfrage und der Antwort auf diese fungiert im Gesprächsverlauf also nur als Nebensequenz (vgl. Peretti 1993: 48).

In Anlehnung an Selting (1987) und Engel (1988: 53) unterscheidet Peretti vier Arten der Verstehensprobleme:

- illokutive, die den Sprechakttyp der Voräußerung oder seine Voraussetzungen betreffen, 
- auditive mit einer weiteren Gliederung in globale und spezifische, wenn die ganze Voräußerung oder Teile von ihr akustisch nicht wahrgenommen werden,

- referentielle, wenn Schwierigkeiten mit dem Bezug der Verweisformen in der Voräußerung auftreten,

- propositionale, wenn der geschilderte Sachverhalt Zweifel hervorruft (vgl. Peretti 1993: 14).

Mit Ausnahme der illokutiven finden wir die übrigen drei Arten in der Gruppe der Verständnisprobleme von Rost-Roth, die die Autorin auf auditives Verstehen, Bedeutungsprobleme und Referenzprobleme (vgl. Rost-Roth 2003: 352) bezieht.

2.1. Auditive Verstehensprobleme treten auf, wenn der Partner Schwierigkeiten hat, die Voräußerung bzw. Teile von ihr akustisch wahrzunehmen. Die Gründe dafür können einerseits bei beiden Partnern liegen wie u. a. undeutliches oder zu schnelles Sprechen, fehlende Aufmerksamkeit, Konzentrationsschwierigkeiten oder Schwerhörigkeit, und andererseits durch Kommunikationsumstände wie Nebengeräusche, Lärm oder andere äußere Störfaktoren bedingt sein (vgl. Peretti 1993: 56). Wird eine Voräußerung als Ganzes nachgefragt, handelt es sich um eine globale auditive Nachfrage. Werden nur Teile der Voräußerung nachgefragt, liegen spezifische auditive Nachfragen vor, die Peretti noch feiner untergliedert in „sequenzspezifische“, „die Gesprächsorganisation“ betreffende und „situationsspezifische“, die „Lärmkulisse“ des Gesprächs betreffende (vgl. Peretti 1993: 56), wobei sich alle diese Abgrenzungen nicht immer deutlich unterscheiden, so dass Überlappungen in Kauf zu nehmen sind (vgl. Beleg 1). Der Kontext und/oder die Situation kann in den meisten Fällen die Erschließung des aktuellen Bezugs solcher Fragen so weit stützen, dass deren Äußerungsform im Deutschen wie im Polnischen gewöhnlich auf die kommunikativ wichtigsten, oft interrogativen Elemente mit eventuellen Erweiterungen verkürzt ist und eine volle Ausformulierung eher zu Ausnahmen gehört.

Globale auditive Nachfragen haben im Deutschen wie im Polnischen vor allem die Form voller bzw. elliptischer routinierter Formulierungen, evtl. in Verbindung mit Höflichkeitsformeln wie im Deutschen Entschuldigung, was haben Sie gesagt?, Entschuldigung, was hast du gesagt?; Wie war das?; Wie bitte?; Bitte?; Was (hast du gesagt/sagst du)? und im Polnischen Przepraszam, co pani powiedziata?; Przepraszam, co powiedziałeś/mówisz?; Stucham?; (Co,) proszę?; Co takiego?; Co (mówisz/ powiedziałaś)?, wobei nur auf das Fragewort allein gekürzte Formeln wenig höflich und alltagssprachlich-salopp wirken (vgl. Engel 1988: 55).

Im Beleg (1) kann das Nichtverstehen durch die Akustik des Telefongesprächs oder durch das lautliche Spiel der kontextuell verbundenen Sprachzeichen Herrn Lange verlangen verursacht sein, die der Gesprächspartner nicht erfasst. Die zweite Deutung geht in der Übersetzung wegen veränderter Sprachzeichen verloren, so dass nur ein nicht näher definiertes Nichtverstehen bleibt. 
(1) (Telefongespräch)
A: Kein Problem. Ich rufe beim Verlag an.
B: Du mußt Herrn Lange verlangen - sagte ich.
A: Bitte? (SW 141)
A: No problem. Zadzwonię do wydawnictwa.
B: W takim razie musisz poprosić do telefonu pana Langa - poradziłam mu.

A: Słucham? (SB 163)

Im Beleg (2) dagegen wird das durch den Außenlärm verursachte akustische Nichtverstehen einzelner Wörter auch spielerisch gezielt zur Humorerzeugung genutzt, was in Wiederholungen der auf phonetischer Ähnlichkeit basierenden Sprachsequenzen sichtbar ist. Nur die erste Rückfrage signalisiert wohl ein rein auditives Nichtverstehen des Gehörten. Als Rückfrage fungieren jeweils die falsch verstandenen Wörter, die in der Antwort korrigiert werden. In der Übersetzung sind beide Funktionen erhalten, allein das Sprachspiel verlangt einen Austausch der einzelnen Wörter durch sinnverwandte mit ähnlicher phonetischer Basis, die das intendierte Sprachspiel ermöglicht.

(2) (Im Frisiersalon, beide Gesprächspartnerinneren sitzen unter der Haube. Die Ausrufezeichen signalisieren die Lautstärke des Gesprächs.)

B: Und wo sind sie jetzt? Bei der Oma? [...]

A: Nein. Der Große ist im Kindergarten, und der Kleine ist bei einer Nachbarin. Ich habe heute einen Maklertermin! - schrie ich.

B: Bitte?

A: Makler!! Ich soll ein HAUS KAUFEN!!

B: Das ist aber eine schöne Aufgabe!

A: Es geht! Ich habe nur noch zehn Tage Zeit dazu! Ich muß noch in DIESEM Jahr das Haus kaufen! Verstehen Sie! Wegen der STEUER!

B: Teuer? Ja, die Häuser in dieser Gegend sind teuer! Wem sagen Sie das!

A: STEUER! FINANZAMT! SCHWARZGELD UNTERBRINGEN!

B: Ach, STEUER! (SW 12)

B: A z kim są teraz? Z babcią? [...]

A: Nie, starszy w przedszkolu, a mały u sąsiadki. Dzisiaj jestem umówiona w biurze nieruchomości! - krzyknęłam.

B: Slucham?

A: Nieruchomości! Mam KUPIĆ DOM!

B: Jakie trudne zadanie!

A: Ujdzie. Mam tylko dziesięć dni czasu! Dom muszę kupić jeszcze w tym roku kalendarzowym. Rozumie pani? Z powodu urzędu finansowego!

B: Finanse? Tak, tak, domy w tej dzielnicy są bardzo drogie! Komu pani to mówi!

A: URZĄD FINANSOWY! UKRYĆ LEWE DOCHODY!

B: Ach, urząd finansowy! (SB 13-14)

Besonders nicht durch Kontext bzw. Situation gestützte Elemente der Voräußerung wie Personennamen, allein stehende Lexeme oder solche, die in der gegebenen 
Konstellation verwirrend wirken, können falsch bzw. nicht wahrgenommen werden und müssen mit spezifischen auditiven Rückfragen in Form von Ergänzungsfragen mit auf dem Fragewort liegender veränderter Intonation nachgefragt werden. Dabei kann das Nichtverstehen auch zusätzliche emotionale Reaktionen wie Ungeduld, Ärger und nicht zuletzt Humor in den literarischen Dialog einbringen. Im Beleg (3) geht in der Übersetzung das Sprachspiel zwischen dem Nachnamen der Heldin Herr und ihrem weiblichen Geschlecht verloren.

(3) A: Franziska Herr. [...]

B: Wie, sagten Sie, war Ihr Name?

A: Mein Name war und ist und bleibt Herr. [So, du dumme Pförtnerpute.] [...]

B: Annegret? Ist eine Frau (Kunstpause) Herr (Räusper, Kunstpause) bei euch angemeldet? (SW 102-103)

A: Franziska Herr. [...]

B: Jak pani godność?

A: Nazywałam się i nadal się nazywam Herr! - [Co za głupia gęś! ] [...]

B: Annegreta? Czy pani (tu sztuczna przerwa) Herr (chrząknięcie, sztuczna przerwa) jest z wami umówiona? (SB 117)

Manche Rückfragen sind allerdings hinsichtlich ihres Bezugs auf den Inhalt oder den Sprechakt undifferenziert und können dem Partner das eine wie das andere sowie lediglich das akustische Nichtverstehen der Voräußerung signalisieren. Beim fehlenden bzw. mangelhaften Kontext oder nicht ausreichenden Vorwissen lassen sich die einzelnen kommunikativen Funktionen der Rück- wie Gegenfrage nicht immer eindeutig erkennen bzw. voneinander trennen. Besonders bei aus einem Wort oder einem parasprachlichen Signal bestehenden Fragen bleibt offen, ob ein auditives oder referentielles Verstehensproblem vorliegt. So kann die Gegenfrage in (4) sowohl die auditive als auch die allgemein referentielle und propositionale Seite betreffen, in (5) dagegen deutet der Kontext eher auf Probleme nicht auditiver Natur hin, was in der polnischen Entsprechung deutlicher wird.

(4) A: Hast du auch den Knopf gedrückt für die kleinere Portion?

B: Hä?! (SW 185)

A: Czy znalazłaś przycisk do mniejszych porcji?

B: Co? (SB 216)

(5) A: Wir haben zu schnell geheiratet. Unsere Tocher war unterwegs.

B: Entspannung und Aufstand?

A: Bitte?

B: Ach, nichts. Bei uns war es ähnlich. (SW 145)

A: Za szybko zapadła decyzja o ślubie. Nasza córeczka była już w drodze.

B: „Rebelia”?

A: Nie rozumiem?

B: Nic nie szkodzi. U nas było podobnie. (SB 216) 
2.2. Referentielle Verstehensprobleme entstehen, wenn der Partner die Voräußerung „nicht zu interpretieren vermag“(Selting 1987: 134), weil er Schwierigkeiten mit deren außersprachlichem Bezug hat. Rost-Roth spricht vor allem dann von Referenzproblemen, ,wenn es dem Hörer z. B. bei lokalen oder temporalen Verweisen nicht möglich ist, die entsprechenden Referenten zu identifizieren" (2003: 355). Die mehrere Bezüge zulassenden problematischen Elemente sind neben lokalen und temporalen Adverbien (13) auch Pronomen $(7-12,14,15)$ und Eigenamen (14), deren richtiger Bezug aus dem situationellen und kontextuellen Gesprächszusammenhang erschlossen werden muss (vgl. Peretti 1993: 57). Mit einer referentiellen Rückfrage signalisiert der Hörer seinem Partner die Mehrdeutigkeit seiner Voräußerung oder eines ihrer Elemente, derer dieser sich selbst vielleicht nicht bewusst ist, weil sein Vorwissen ihn die Situation anders einschätzen lässt. Solche Nachfragen haben in beiden Sprachen meist die Form verbloser mit Fragewort eingeleiteter Fragestrukturen, in denen das problematische Element oder die gemeinte Referenz genannt wird (6-14). Volle Fragestrukturen sind seltener und können die Form von Ergänzungs- oder Entscheidungsfragen mit Zweitstellung des Finitums im Deutschen $(9,13,15)$ haben. Der Akzent liegt jeweils auf dem Fragewort oder auf dem nachgefragten Element, was im gedruckten Text ggf. die Schriftart signalisiert $(7,12,14,15)$. Bei Anredeforme(1)n und deiktischen Formen kommt es zu deren Wechsel (mein $\rightarrow$ dein $\|$ mój $\rightarrow$ twój, wir $\rightarrow$ Sie $\|$ my $\rightarrow$ pan/pani, vgl. 8, 9, 12, 14, 15). Zuweilen können die Fragestrukturen um metasprachliche oder kommentierende Ausdrücke erweitert sein $(11,10)$. Wegen der reduzierten Fragestruktur lassen sich solche Reparatursequenzen im Polnischen relativ problemlos wiedergeben. Ihr Beitrag zur Wirkung des literarischen Textes besteht im regen Wort- und Zuordnungswechsel, der den Dialogen Lebhaftigkeit und Witz verleiht. Das Wissen des Lesers erlaubt es ihm, sich an dem so entstandenen Situationshumor zu erfreuen und Empathie für die Romanfiguren und deren Verhalten zu entwickeln.

(6) A: Können Sie morgen in mein Büro kommen?

[Solange wollte ich nicht mehr warten. Ich hatte JETZT Lust auf Herrn Dr. Winkel und seinen Fünf-Millionen-Dollar-Gewinn. Ich sah auf die Uhr. Mitternacht.]

B: Haben Sie Lust auf ein Glas Wein?

A: Bitte?

B: Ich meine, ob Sie es einrichten können, die Sprechstunde jetzt gleich abzuhalten? Bei mir zu Hause. Die Kinder schlafen. Es wäre gerade günstig.

A: Ja - sagte er - das ließe sich einrichten. (SW 44)

A: Czy mogłaby pani przyjść jutro do mojej kancelarii?

[Nie miałam ochoty czekać do jutra. Miałam ochotę TERAZ porozmawiać z panem doktorem Winklem na temat dodatkowego dochodu w wysokości pięciu milionów dolarów. Zerknęłam na zegarek. Północ.]

B. Czy nie ma pan ochoty na kieliszek wina?

A: Slucham?

B: Pytam, czy nie mógłby pan odbyć swoich godzin przyjęć teraz? U mnie w domu? Dzieci śpią. To korzystna pora. 
A: [Tak - odpart - to dałoby się zrobić. - Die Antwort ist in der Übersetzung ausgelassen.] (SB 51)

(7) A: Und nun zu Ihrem anderen Anliegen - sagte Herr Winkel geduldig.

B: Welches ANDERE Anliegen?

A: Sie wollen eine Immobilie erwerben.

B: Klar - lallte ich - das ist mein Begehr. (SW 19)

A: A teraz porozmawiajmy o pani drugiej sprawie - powiedział pan Winkel cierpliwie.

B: Jaka DRUGA sprawa?

A: Przecież pani chciała kupić nieruchomość.

B: Jasne - wyrwało mi się bełkotliwie - Takie jest moje życzenie. (SB 21)

(8) A: Aber die Scheidung schien mir doch vorrangig zu sein.

B: Welche Scheidung? - fragte ich überrascht.

A: Ihre Scheidung - sagte Herr Winkel. (SW 19)

A: Ale rozwód wydawał mi się ważniejszy.

B: Jaki rozwód? - zapytałam zaskoczona.

A: Pani rozwód - odparł adwokat. (SB 22)

(9) A: Na, wenn das kein Zufall ist! Wir haben eben von Ihnen gesprochen!

B: Wer, wir? - fragte ich.

A: Na, Enno und ich! (SW 23)

A: No coś takiego, co za przypadek! Właśnie o pani mówiliśmy!

B: To znaczy kto? [spytałam;auch möglich: Kto (to jest), my?]

A: No, Enno i ja. (SB 26)

(10) A: Unser Geld.

B: [Wilhelm lachte Hohn.] Unser Geld? Daß ich nicht lache! Wo hast du denn in den letzten fünf Jahren Geld verdient, wenn ich mal fragen darf? (SW 134)

A: Nasze pieniądze.

B: [Wilhelm roześmiał się szyderczo.] Nasze pieniądze? Nie rozśmieszaj mnie! A w jaki to sposób ty zarabiałaś, siedząc przez ostatnie pięć lat w domu, jeśli wolno zapytać? (SB 155)

(11) A: Enno, bitte teile Herrn Großkötter doch mit, daß ich erstens nicht bereit bin, mit ihm zusammenzuziehen, und daß ich mir doch noch sehr überlegen werde, ob ich mit ihm zusammen das Drehbuch schreibe - sagte ich [...]

B: Welches Drehbuch? fragten Wilhelm und Enno gleichzeitig. (SW 142)

A: Enno, poinformuj z łaski swojej pana Grosskoettera, że nie jestem gotowa zamieszkać razem z nim - to po pierwsze, a po drugie, że się jeszcze zastanowię, czy razem z nim napiszę scenariusz - dodałam $[\ldots]$

B: Jaki scenariusz? - zapytali równocześnie. (SB 164)

(12) A: Außerdem, woher wissen die meine neue Adresse? - fragte Wilhelm mehr sich als mich.

B: Wieso DEINE Adresse? - entgegnete ich ratlos. - Das ist MEINE Adresse, und das Päckchen ist für MICH. (SW 138)

A: A skąd oni mają mój nowy adres? - Wilhelm zadał to pytanie raczej sobie niż mnie.

B: Jak to TWÓJ adres? - zareagowałam dość bezradnie. - To jest MÓJ adres, a paczka jest dla MNIE. (SB 159)

(13) A: Hätte nicht gedacht, daß die so schnell schicken. - sagte Wilhelm [...].

B: Wie, die schicken so schnell? - [Gott, was mußte der arme Kerl Gehirnzellen in der Karibik gelassen haben!]

A: Wieso schicken die mir'ne ganze Kiste? Ich wollte doch nur ein Exemplar!

B: Wie, du wolltest nur ein Exemplar? - fragte ich ihn [...]. (SW 138-139)

A: Nie sądziłem, że tak szybko je przyślą - powiedział Wilhelm [...]. 
B: Jak to, przysłali tak szybko? - [Boże, ten biedny człowiek odmóżdżył się całkowicie na tych Karaibach.]

A: Dlaczego przysłali całą paczkę? Przecież zamówiłem tylko jeden egzemplarz.

B: [Jak to,] Chcesz tylko jeden egzemplarz? - zapytałam [...]. (SB 159-160)

(14) A: Franziska, das ist Gerd.

B: Welcher Gerd?

A: Mein Mann. [Ich verschluckte mich fast an einem Popkornkrümel.]

B: Dein MANN? Nein.

A: Doch. Krieg ich jetzt die Kündigung?

B: Nein! Ich meine... Du bist verheiratet? Warum hast du mir das nie gesagt? (SW 389)

A: Franzisko, to jest Gerhard.

B: Co za Gerhard?

A: Mój mąż. [Niemal udławiłam się popkornem.]

B: Twój MĄŻ? Nie!

A: A jednak! Czy teraz mnie zwolnisz?

B: Nie! To znaczy... Jesteś zamężna? Dlaczego nigdy mi o tym nie powiedziałaś? (SB 462)

(15) A: Eine knappe Million, flüsterte ich dann.

B: Davon kriegen wir auf jeden Fall drei Siebtel, sagte er sachlich ...

A: SIE kriegen drei Siebtel? - fragte ich entrüstet.

B: SIE! Wenn ich „wir“ sage, meine ich „Sie“! lächelte der Anwalt gönnerhaft. (SW 15-16)

A: Prawie pół miliona - szepnęłam.

B: Z tej sumy na pewno otrzymamy trzy siódme! rzekł rzeczowym tonem.

A: Pan otrzyma trzy siódme? - zapytałam oburzona.

B: PANI! Gdy mówię „my”, mam na myśli panią! - adwokat uśmiechał się protekcjonalnie. (SB 17)

2.3. „Um Bedeutungsverstehensprobleme handelt es sich, wenn die Äußerung zwar auditiv wahrgenommen, aber Elementen der Ausdrucksseite kein inhaltlicher Sinn zugeordnet werden kann" (Rost-Roth 2003: 353), so dass die Situation für den Partner unklar bleibt. Auch die ganze Mitteilung kann dabei inhaltlich als unverständlich bzw. nicht hinreichend präzise empfunden werden. Ein solches Nichtverstehen ruft zudem meist Staunen, Verwunderung oder Zweifel am Gehörten und Verstandenen hervor (20, 21, 23). Nachgefragt wird mit (teilweiser) Wiederholung der Mitteilung in nachdrücklicher Frageintonation $(16,19,20,21)$, mit zur Klärung des Gesagten auffordernden Fragen mit Verba sentiendi $(17,18)$, mit auf die ganze Voräußerung bezogenen einzelnen Fragewörtern (19) oder mit routinierten Formulierungen wie Na und?, Und? \|No i co? (No) i co z tego? (22). Als Antwort auf solche Rück- und Gegenfragen (21) folgt meist eine Bestätigung mit wiederholter Bezugsäußerung (16) oder eine neue, präzisierende und damit die bestehenden Zweifel beseitigende Formulierung (17, 19, 20, 21). Einer solchen Präzisierung bedürfen vor allem auf ganze Sachverhalte verweisende Pronomina wie es $\|$ to, deren Referenz bei jedem Gesprächspartner anders sein kann (21).

(16) A: Ich habe natürlich schon dort angerufen - sagte Enno.

B: Und?

A: Sie wollen es drucken.

B: Sie wollen es drucken? 
A: Ja. Sie wollen es drucken. (SW 97)

A: Już do nich dzwoniłem - odparł Enno.

B: No i co?

A: Chcą to wydać.

B: CHCĄ TO WYDAĆ?

A: Tak. Chcą to wydać. (SB 111)

(17) A: Du bist jetzt eine Person des öffentlichen Lebens - sagte Enno. - Du lebst gefährlich.

B: Wie meinst du das?

A: Wir sollten eine Alarmanlage einbauen lassen. (SW 264)

A: Jesteś teraz znaną osobistością - stwierdził natomiast. - To niebezpieczne.

B: Co masz na myśli?

A: Powinnaś zainstalować tu alarm. (SB 310)

(18) A: Paula, rief ich. Sie können natürlich jetzt gehen!

B: Meinen Sie, ich lasse Sie jetzt im Stich? - fragte Paula zurück. (SW 205)

A: Pani Paulo, pani, naturalnie, może już iść!

B: Czy Pani sądzi, że zostawię ją w takiej sytuacji? - odpowiedziała pytaniem na pytanie. (SB 240) [Änderungsvorschlag: odpowiedziała Paula pytaniem]

(19) A: Was machen die Kinder?

B: Sie spielen draußen im Sand - sagte Paula. - Alles bestens. Der Keller stand unter Wasser. Aber Enno und ich haben das erledigt.

A: WAAS?

B: Enno hat mir über das Funktelefon Anweisungen gegeben, [...] (SW 233)

A: A co u dzieci?

B: Bawią się na dworze. Wszystko w porządku. Piwnica była zalana wodą. Ale Enno i ja poradziliśmy sobie.

A: Coo?

B: Enno dawał mi wskazówki przez telefon komórkowy, [...] (SB 273)

(20) [Dann ließ Enno Winkel die Bombe platzen.]

A: Sie hat deine Dings, deine ... Ehegeschichte bei einem Verlag eingereicht.

[Ich starrte ihn an.]

B: Sie hat WAS? (SW 95)

[I wtedy Enno wreszcie puścił parę z ust.]

A: Ona wysłała te twoje zapiski, eee, historię twojego małżeństwa... do wydawnictwa. [Popatrzyłam na niego w osłupieniu.]

B: CO zrobila? (109)

(21) [Ich kicherte. Enno Winkel mißdeutete das.]

A: Nicht wahr, Sie spüren es auch? - sagte er, meinen Unterarm streifend.

B: Ich spüre WAS auch? - fragte ich mit herausfordernder Vorfreude. Los, mach doch!

A: Daß wir uns mögen - gurrte Enno. (SW 47)

[Zachichotałam. Enno Winkel zrozumiał to opacznie.]

A: Pani doskonale sama o tym wie, nieprawdaż? - powiedział, głaszcząc moją rękę.

B: O CZYM wiem? - zapytałam zaczepnie, już z góry się ciesząc. No, zaczynaj!

A: O tym, że się lubimy - gruchał Enno. (SB 54)

(22) A: Wir tun beide, was wir gut können und was uns Spaß macht. [Sie lächelte entwaffnend.]

B: Hast du aber doch nicht nötig - sagte ich.

A: Na und? Du hast es auch nicht nötig, Bücher zu schreiben und Filme zu machen und auf Lesereisen zu gehen [...]. (SW 221)

A: Obie robimy to, co potrafimy i co lubimy - [Paula uśmiechnęła się rozbrajająco.] 
B: Przecież ty naprawdę nie musisz! - powtórzyłam.

A: No i co z tego? Ty też nie musisz pisać książek ani scenariuszy do filmów, spotykać się z czytelnikami [...]. (SB 259)

Im Beleg (18) ist die Reaktion der Gesprächspartnerin im kommentierenden Text zusätzlich als Rückfrage beschrieben. Allerdings liegt in der polnischen Übersetzung wohl eine Überinterpretation vor, denn die Voräußerung ist eindeutig eine Aufforderung, auf die auch mit einer Nichtverstehen signalisierenden Rückfrage reagiert werden kann. Die angeführten Belege zeigen, dass Bedeutungsprobleme auch emotionale Reaktionen wie Staunen, Verwunderung, Überraschung, Zweifel hervorrufen und in der Konsequenz zu Nachfragen führen.

Im nachfolgenden Beleg (23) wird ähnlich wie in (3) ein allgemeines Nichtverstehen inszeniert, jedoch versucht die Gesprächspartnerin die Situation auch sprachlich zu klären, indem sie gezielt um eine Erklärung bittet und damit - literarisch gesehen - die das Sprachspiel auslösenden Lexeme Herr (als Anredeform und als Nachname) und Frau (nur Anredeform) in direkten Zusammenhang bringt. So dient hier die Rückfrage ebenfalls zur Erzeugung der Situations- und Sprachkomik (ähnlich wie in 13), die durch verschiedenes Vorwissen der Gesprächspartner und das Sprachspiel der gleichlautenden Anredeform und des Nachnamens zustande kommt. Das Staunen hervorrufende Nichtverstehen der nachfragenden Gesprächspartnerin wird in der Beschreibung zusätzlich hervorgehoben. In der Übersetzung bleibt die Situationskomik erhalten, das Sprachspiel aber geht zum Teil verloren.

(23) A: Sagen Sie, Frau ... Großkötter ...

B: Herr-Großkötter! - sagte ich.

A: Bitte? - Die Dame betrachtete mich mit äußerstem Erstaunen.

B: Herr-Großkötter - beharrte ich trotzig. [...]

A: Wieso Herr Großkötter, wenn ich Frau Großkötter sage? - entfuhr es ihr.

B: Frau Herr-Großkötter - sagte ich. -Ist das denn so schwer?! (SW 51-52)

A: Niech mi pani powie, pani Grosskoetter ...

B: Herr-Grosskoetter - poprawiłam.

A: Słucham? - madame patrzyła na mnie $\mathbf{z}$ największym zdumieniem.

B: Herr-Grosskoetter - powtórzyłam z naciskiem. [...]

A: Dlaczego Herr Grosskoetter, skoro ja mówię „pani Grosskoetter“? - indagowała.

B: Pani Herr-Grosskoetter - powtórzyłam i dodałam: Czy to takie trudne? (SB 59)

Die manchen Rückfragen beigefügten Kommentare versprachlichen und verdeutlichen somit im literarischen Dialog die Reaktionen der Fragenden, die man sonst im Gespräch der Intonation, dem Tonfall und dem mimisch-gestischen Verhalten des Partners entnehmen kann, wie Staunen, Verwunderung, Überraschung und Zweifel, wenn der Fragende den satzsemantischen Gehalt der Voräußerung in sein Vorwissen nicht einordnen kann oder diesen in einer solchen Form nicht erwartet hat (vgl. 19, 20, 21, 23). Peretti rechnet solche Nachfragen propositionalen Rückfragen zu, die ,primär 
der routinierten Vergewisserung [dienen], bei der es sich weniger um das Verstehensdefizit als um eine Anzeige von Aufmerksamkeit oder Kooperationsbereitschaft dem Gesprächspartner gegenüber handelt" (Peretti 1993: 63). Sie stehen damit den Vergewisserungsfragen nahe, die ebenfalls zur Informations- und Verständnissicherung gestellt werden. Ähnliche Überlappungsprobleme bei der Klassifizierung stellt auch Rost-Roth fest, indem sie schreibt:

Handelt es sich um Nachfragen in Form von Wiederholungen, unterscheiden sich diese im Prinzip nicht von verständnissichernden Nachfragen bei auditiven Problemen. Eine Klassifizierung als (,auditives ') Verstehensproblem oder als ,Vergewisserung/Informationssicherung' kann also nicht aus der Nachfrageformulierung, sondern nur über Kontextwissen abgeleitet werden. (Rost-Roth 2003: 362).

\section{3.}

Vergewisserungsfragen können demnach sowohl die Form als auch den Inhalt der Voräußerung betreffen. Besonders in kontextarmen unter schwierigen oder störenden akustischen Bedingungen geführten Gesprächen sind beide Bezüge möglich. Die Nachfrage kann demnach eine allgemeine konventionelle Formulierung (Wie) Bitte? || Stucham? Proszę? oder eine spezifische durch die Voräußerung bedingte Form haben, ähnlich wie bei allen Verstehensproblemen. „Der Bezug auf die Ausdrucksseite erfolgt mit Wiederholungen, der Bezug auf die Inhaltsseite mit Paraphrasierungen oder anderen Reformulierungen als Explikation von Interpretationen“" (Rost-Roth 2003: 363). Das Telefongespräch in Beleg (24) basiert auf einem solchen Spiel zwischen lautlichem Nichtverstehen (Bitte?/Stucham?) und Zweifel an der inhaltlichen Richtigkeit des Gehörten, das die Verwendung der Synonyme verdeutlicht (Jetzt, sofort? || Teraz? Zaraz?):

(24) A: Du, Viktor... ich komme jetzt.

B: ...bitte...?

A: Ich komme jetzt nach Hamburg.

B: Jetzt, sofort?

A: Ja! (SW 145)

A: Wiktorze, ja zaraz przyjadę.

B: ...slucham...?

A: Przyjadę zaraz do Hamburga.

B: Teraz? Zaraz?

A: Tak! (SB 164)

In inhaltsbezogenen Vergewisserungsfragen wiederholt der Fragende das konkrete Element allein oder mit einem zusätzlichen, die Ungewissheit signalisierenden Kommentar, um Gewissheit für sein eventuelles weiteres Handeln zu gewinnen. In der Übersetzung wurde der Ortsname „Schröpfingen“, durch eine allgemeine Ortsbe- 
zeichnung mit erläuterndem Attribut „wieś, gdzieś na dole“ ersetzt, was für den Leser des übersetzten Textes vielleicht verständlicher ist.

(25) [Als es vorbei war, blieben wir noch lange sitzen.]

A: Du?

B: Bitte sagen Sie jetzt nichts.

A: Weißt du, wo mein Turnschuh ist?

B: In Schröpfingen. Vielleicht.

A: Schröpfingen? Das find ich. (SW 252)

[Potem siedzieliśmy na łące i długo rozmawialiśmy.]

A: Słuchaj?

B: Proszę, niech pan nic nie mówi.

A: Czy wiesz może, gdzie jest mój trampek?

B: We wsi, gdzieś na dole...

A: We wsi? Na dole? To na pewno go znajdę. (SB 297)

(26) A: Ich gehe jeden Tag um halb drei nach Hause.

B: Jeden Tag?

A: Ausnahmen bestätigen die Regel. (SW 177)

A: Podejmę się pracy u pani, pod warunkiem, że [codziennie] o wpół do trzeciej już będę wolna.

B: Codziennie?

A: Wyjątki potwierdzają regułę. (SB 206)

(27) A: Das gibt bestimmt einen irren Effekt, besonders, wenn sie mitten in der Nacht auf uns herunterkrachen.

B: [Enno packte mich begeistert an den Schultern.] Uns? Hast du UNS gesagt?

A: [...] Enno, sagte ich, um Fassung ringend. - Ich habe einen Scherz gemacht.

B: Aber du hast UNS gesagt - sagte Enno glücklich. (SW 127)

A: Jeśli kiedyś spadną na nas nocą, efekt będzie porażający.

B: [Enno chwycił mnie za ręce.] Nas? Powiedziałaś: NAS?

A: [...] Enno, to był żart - wyjaśniłam, usiłując się opanować.

B: Ale ty przecież powiedziałaś NAS - Enno był uszczęśliwiony. (SB 147)

Einer Vergewisserungsfrage kann auch eine weitere Frage folgen, die die Nachfrage begründet. Die Sequenz aus einer Vergewisserungsfrage mit veränderter Wortfolge und Intonation und der darauf folgenden Ergänzungsfrage verleiht dem Gespräch Emotionalität (Entrüstung, Empörung, Zweifel), was die verwendeten Abtönungspartikeln denn, etwa $\|$ niby, jakiż, aby verdeutlichen wie in (28-30):

(28) A: Einer musste ja das Geld verdienen! Im Schweiße meines Angesichts! Und wie dankst du es mir?

B: ICH - DIR - DANKEN? Wofür denn? Daß du mich GESCHWÄNGERT hast? (SW 215)

A: Ktoś przecież musiał zarabić na życie! W pocie czoła! I jak mi teraz za to dziękujesz?

B: JA TOBIE MAM DZIĘKOWAĆ? A niby za co? Żeś mnie zapłodnił? (SB 253)

(29) A: Probleme? - Paula hörte mit dem Teigkneten auf.

B: Er will den Titel ändern - brachte ich mühsam hervor.

A: Den Titel „Ehelos glücklich“ will er ändern? In was denn? (SW 210)

A: Kłopoty? - [Paula] na chwilę przestała wyrabiać ciasto. 
B: Chce zmienić tytuł -wyrzuciłam z siebie z trudem.

A: [Tytul , Szczęśliwa niezamężna” chce zmienić?] Na jaki? [Vorschlag: Na jakiż to?] SB 247)

(30) A: Stellen Sie sich vor, ich gehe bei jedem Wind und Wetter mit den Kindern quer durch die Stadt zu Fuß, nur um in den Stadtwald zu kommen.

B: Mit den Kindern? Überfordern Sie die nicht etwas?

A: Ich schieb den einen im Buggy und den anderen auf einem Dreirad vor mir her - gab ich errötend zu. (SW 17)

A: Niech pan sobie wyobrazi, że chodzę z dziećmi do lasku miejskiego pieszo, przez całe miasto! I to przy każdej pogodzie!

B: $\mathbf{Z}$ dziećmi? Czy to aby nie za duży wysilek dla nich?

A: Jeden jedzie w składanym wózku, drugiego pcham na rowerku trójkołowym - przyznałam, rumieniąc się. (SB 19)

Die sprachlichen Formulierungsmittel von Vergewisserungsfragen sind in beiden Sprachen vergleichbar und verdeutlichen die Wiederholung in der Nachfrage der in der Voräußerung angezweifelten Elemente. In der Übersetzung kann das zuweilen verloren gehen, wenn der pragmatische Aspekt der Wiederholungen nicht richtig erkannt und auf diese verzichtet wird (vgl. 26, 29), obwohl sie sich problemlos wiedergeben ließen.

Vergewisserungsfragen können sich in ihrem Wortlaut von der Voräußerung unterscheiden, wenn mit ihnen die eigene Interpretation des Fragenden oder dessen emotionale Reaktion ausgedrückt wird:

(31) A: Dagegen müssen wir angehen.

B: Du meinst, rechtlich? (SW 156)

A: Musimy tu coś zaradzić.

B: Masz na myśli przepisy? (SB 181)

(32) A: Und hast du auf Anhieb ihren Namen verstanden? -wunderte ich mich.

B: Nein - sagte Paula - aber ich habe ihn mir buchstabieren lassen.

A: Tatsächlich? - Ich staunte.

B: Das habe ich mir bei Tante Trautschn so gewöhnt - sagte Paula. (SW 232)

A: I od razu zrozumiałaś, jak się nazywa? [zdziwitam się]

B: Nie, [odparła Paula] poprosiłam, aby mi przeliterowała nazwisko.

A: Naprawdę? - to mi zaimponowało.

B: Nauczyłam się tego, będąc u cioteczki Trautschn. [powiedziała Paula] (SB 272)

Mit Gegenfragen als Reaktion auf eine gestellte Frage signalisiert der Gesprächspartner meist seine auf die Zweckmäßigkeit der gestellten Frage bezogene Unsicherheit und erst dann deren Inhalt:

(33) A: Wohin fahren Sie denn?

B: Nach Hamburg, ...

A: Fahren Sie da über Stuttgart? - testete ich vorsichtig an.

$\mathrm{B}$ : Wieso? Is des hier net rischdisch?

A: Also dieser Zug fährt nach Süden - sagte ich. Und Hamburg liegt im Norden. (SW 226)

A: Dokąd pani jedzie? 
B: Do Hamburga ...

A: I jedzie pani przez Stuttgart?

B: A bo co? Coś nie tak?

A: No wie pani, Hamburg jest na północy, a ten pociąg jedzie w kierunku południowym - wyjaśniłam. (SB 265)

\section{4.}

Nachfragen zu Erwartungsproblemen sondert nur Rost-Roth als selbständige Art aus und bezieht sie auf solche, die Informationslücken der Voräußerung signalisieren (vgl. Rost-Roth 2003: 361), so dass diese mit dem Vorwissen und den daraus resultierenden Erwartungen des Gesprächspartners nicht übereinstimmt. Das nachgefragte Element bzw. die ganze Phrase wird wiederholt und prosodisch exponiert. Die Erwartung kann allerdings entweder die Referenz oder die Bedeutung betreffen, womit solche Nachfragen ebenfalls in die Nähe der Vergewisserungsfrage treten, was Rost-Roth (2003: 362) selbst zugibt. Die Unterscheidung beider Arten beruht eher auf der Interpretation und dem Kontext der nachfolgenden Antwort. Eine solche Nachfrage kann dadurch eine Aufforderung zur Weiterführung des Gesprächs und zum Preisgeben weiterer Informationen sein (37) und somit an der Grenze zu Nachfragen mit diversen anderen kommunikativen Funktionen liegen (vgl. 35).

(34) A: Für uns drei wird es reichen - antwortete ich unverbindlich.

B: Wie - für uns drei? Sind wir nicht vier? [Wilhelm schien ernste Koordinationsprobleme zu haben.] (SW 133)

A: Dla naszej trójki wystarczy - odpowiedziałam mimochodem.

B: Dla naszej trójki? Przecież jest nas czworo. [Wilhelm wydawał się mieć problemy z koordynacją.] (SB 154)

(35) [Dazu legte ich ein Bild von den Kindern mit den Zeilen: Danke, liebe Paula, daß es dich gibt.]

A: Dich? - fragte Paula.

B: Na klar - sagte ich - oder glauben Sie, die Kinder würden Sie siezen?

A: Dann möchte ich aber auch, daß wir „du“ zueinander sagen - sagte Paula. (SW 218)

[Do niej dołożyłam zdjęcie chłopców z dedykacją: Dziękujemy Ci, kochana Paulo, za to, że jesteś.]

A: Że jesteś? -zdziwiła się Paula.

B: No jasne - odparłam. Czy pani sądzi, że dzieci napisałyby „pani”?

A: W takim razie proponuję, abyśmy też przeszły na „ty” - zaproponowała Paula. (SB 256)

(36) A: Enno, [...] du redest die ganze Zeit in diesem Ton! Ist es da verwunderlich, wenn ich mich mal verplappere?

B: Du willst also nicht, daß ich mit dir hier einziehe?

A: Dazu besteht überhaupt kein Grund! Du wohnst doch direkt gegenüber. (SW 127)

A: Enno, [...] cały czas mówisz w tym tonie! Czy to dziwne, że w takiej sytuacji się przejęzyczyłam?

B: A więc nie chcesz, żebym się tu wprowadzil?

A: Nie ma ku temu powodu! Mieszkasz przecież naprzeciwko. (SB 147)

(37) A: Franziska! -schrie sie begeistert. - Ich hab schon soviel von dir gehört!

B: Ja? - fragte ich erstaunt. - Von wem denn? 
A: Von Wilhelm natürlich! (SW 328)

A: Franzisko! - zawołała rozentuzjazmowana. - Już tyle o tobie słyszałam!

B: Tak? - zapytałam zdziwiona. - Od kogo?

A: Naturalnie od Wilhelma. (SB 387)

\section{5.}

Als vierte Art unterscheidet Rost-Roth (2003: 363) allgemeine Nachfragen mit anderen Funktionen als den bisher genannten. Dazu gehören vor allem die bereits unter 1 . erwähnten Nachfragen zum Zeitgewinn. Im Kontext einer Voräußerung können solche Fragen als Kontaktsignal fungieren (vgl. Engel 1988: 58). Der Gesprächspartner signalisiert auf diese Weise, dass er zuhört bzw. die Voräußerung wahrgenommen hat (38, 39). Er kann damit seine Aufmerksamkeit und Kontaktbereitschaft bekunden (40, 41), den Partner korrigieren wollen (35) oder ihn lediglich zum Weitersprechen ermuntern (42), was die angeschlossenen Sequenzen oft verdeutlichen. Es können auch Höflichkeitsgründe vorliegen (41). Solche Nachfragen haben in beiden Sprachen gewöhnlich konventionalisierte Formen, so dass ihre Wiedergabe keine Probleme bereitet.

(38) A: Enno?! [Enno fuhr geradeaus. Seine Hand hatte er ruckartig aus meinem Nacken entfernt.]

B: Hm?

A: Es tut mir leid, Entschuldigung! (SW 116)

A: Enno? [Enno jechał spokojnie. Po chwili zabrał rękę z mojego karku.]

B: No?

A: Przepraszam, przykro mi. (SB 133)

(39) A: O. K. - sagte mein Anwalt und drückte mir fest die Hand.

B: Du, Enno?

A: Ja?

B Darf ich dich noch um etwas bitten?

A: Natürlich. (SW 100)

A: OK - powiedział mój mecenas i mocno ścisnął mi dłoń.

B: Enno...?

A: Tak, słucham?

B: Czy mogę cię o coś poprosić?

A: Naturalnie. (SB 115)

(40) A: Frau Herr-Großkötter?

B: (Ich sprang auf.) Ja?

A: Kommen Sie bitte weiter! Herr Dr. Winkel erwartet Sie! (SW 14)

A: Pani Herr-Grosskoetter? [ausgelassen in der Übersetzung: B: (Szybko wstałam z krzesła.) Tak?] Proszę wejść. Doktor Winkel czeka na panią. (SB 15)

(41) A: Hallo? Wer bedient hier?!

B: Kann ich Ihnen helfen? - ein nettes, blasses Mädchen mit Brille kam dienstfertig herbei. (SW 154)

A: Halo, czy tu ktoś obsługuje?

B: W czym mogę pani pomóc? - miła, bladziutka dziewczyna w okularach podbiegła do mnie natychmiast. (SB 178) 
(42) A: Es ist ganz O. K. so - sagte ich - reg dich nicht auf. Der Lektor ist für mich zuständig, nicht der Geschäftsführer.

B: Und? Erzähl doch mal. Was habt ihr besprochen?! (SW 113-114)

A: Wszystko w porządku, nie denerwuj się. Zajmuje się mną redaktor, a nie dyrektor wydawnictwa. wyjaśniłam.

B: Hm? No, opowiadaj! Coście ustalili? (SB 130-131)

$\mathrm{Zu}$ dieser Gruppe zählen auch einen Gegenvorschlag enthaltende Gegenfragen in Form von Entscheidungs- oder Ergänzungsfragen, die zusätzlich mit $U n d \| A$ als alternativem Konjunktor (vgl. Engel u. a. 1999: 1148) eingeleitet sein können.

(43) A: Karibik - sagte Will Groß.

B: Aha - sagte ich. - Willste 'n Bier?

A: Schampus haste nicht? (SW 132)

A: Karaiby - rzucił Wilhelm Wielki.

B: Aha - skwitowałam. - Napijesz się piwa?

A: Nie masz szampana? (SB 153)

\section{6.}

Die vorgenommene Analyse zeigt, dass das Erkennen der Funktion bei vielen Nachfragen problematisch und vor allem vom Kontext und dem Vorwissen der Gesprächspartner abhängig ist. Rost-Roth stellt am Ende ihrer Untersuchung auch fest,

daß es im konkreten Einzelfall oft nicht möglich ist, eindeutige Zuordnungen zu bestimmten Nachfragefunktionen vorzunehmen. [...] Methodisch wird in den meisten Untersuchungen so verfahren, daß nicht nur die Nachfrage selbst, sondern auch die Antwortreaktion daraufhin betrachtet wird, welcher Art die Problembehandlung ist, um über (erfolgreiche) Problembehandlungen auf die Problemursache zu schließen. (Rost-Roth 2003: 368).

Je genauer die Aufgliederung in die einzelnen Funktionsarten ist, desto schwieriger ist es, den Grund für die konkrete Nachfrage zu bestimmen und die einzelnen Funktionen zu unterscheiden. In der direkten Kommunikation können die intonatorischen und gestisch-mimischen Mittel die Unterscheidung erleichtern. In geschriebenen literarischen Dialogen unterstützen eine solche Unterscheidung die beigefügten Kommentare, welche Intonation, Körperverhalten und vor allem den Kontext erläutern, der in den meisten Fällen für Verstehensprobleme aller Art ausschlaggebend ist. Eine genaue Differenzierung der Funktionen ist dagegen vor allem für pragmatische Analysen notwendig, was im Kontext literarischer Werke von Bedeutung ist, da „,von der Übersetzung die Bewahrung der pragmatischen Elemente der Vorlage [erwartet] wird“ (Krysztofiak 2013: 36). In literarischen Dialogen dienen Rück- und Gegenfragen mit den ihnen eventuell angefügten Kommentaren zur Herstellung natürlicher kommunikativer Situationen, zum Beleben der Handlung und zur Vermittlung verschiedener 
Emotionen der Gesprächspartner. Darüber hinaus werden sie gezielt eingesetzt, um Situations- und Sprachkomik hervorzurufen und so die Wirkung des literarischen Textes zu erhöhen. Die Aufgabe des Übersetzers besteht hier darin, diese Funktionen zu erkennen und sie der Intention des Autors gemäß wiederzugeben. Ihre sprachliche Realisierung ist dabei nicht nur vom jeweiligen dialogischen Kontext, sondern auch von ihrer pragmatischen und ästhetischen Textfunktion abhängig. Im Falle der Sprachkomik und vor allem des Komik hervorrufenden Sprachspiels ist das pragmatisch nicht immer einfach und möglich, denn ,der pragmatische Aspekt betrifft nicht nur die Treue einer jeden Übersetzung gegenüber dem Original, sondern auch die Treue der Übersetzung in der intersemiotischen Perspektive, wobei im Hinblick auf die literarische Übersetzung die ästhetische Dimension der Treue miteinbezogen wird“" (Krysztofiak 2013: 36).

\section{Quellennachweis}

Hera Lind: Das Superweib. Frankfurt am Main: Fischer Taschenbuch Verlag, 1994. (= SW)

Hera Lind: Superbaba. Übersetzt von Janina Zapaśnik-Ogrzewalska. Warszawa: Przedsiębiorstwo Naukowo-Techniczne CIBET, 1997. (= SB)

\section{Literatur}

Altmann, Hans Peter (1987): „Zur Problematik der Konstitution von Satzmodi als Formtypen.“ In: Maibauer, Jörg (Hg.): Satzmodus zwischen Grammatik und Pragmatik. Tübingen: Max Niemeyer Verlag. 22-56.

Darski, Józef (2004): Linguistisches Analysemodell. Definitionen grundlegender grammatischer Begriffe. Poznań: Wydawnictwo Naukowe UAM.

Engel, Ulrich (1988): Deutsche Grammatik. Heidelberg: Judicium Verlag.

Engel, Ulrich ( $\left.{ }^{4} 2009\right)$ : Syntax der deutschen Gegenwartssprache. Berlin: Erich Schmidt Verlag.

Engel, Ulrich u. a. (1999): Deutsch-polnische kontrastive Grammatik. Heidelberg: Julius Groos Verlag.

Helbig, Gerhard/Buscha, Joachim ( ${ }^{152001): ~ D e u t s c h e ~ G r a m m a t i k . ~ E i n ~ H a n d b u c h ~ f u ̈ r ~ d e n ~ A u s l a ̈ n d e r u n t e r-~}$ richt. Leipzig u. a.: Langenscheidt Verlag.

Kallmeyer, Werner (1977): „Verständigungsprobleme in Alltagsgesprächen.“ Der Deutschunterricht, 52-68. Krysztofiak, Maria (2013): Einführung in die Übersetzungskultur. Frankfurt a. M. u. a.: Peter Lang Verlag. Maibauer, Jörg (1987a): „Zu Form und Funktion von Echofragen.“ In: Rosengren, Inger (Hg.): Sprache und Pragmatik. Lunder Symposium 1986. Stockholm: Almqvist \& Wiksell. 335-365.

Maibauer, Jörg (Hg.) (1987b): Satzmodus zwischen Grammatik und Pragmatik. Tübingen: Max Niemeyer Verlag.

Nagórko, Alicja (2010): Podręczna gramatyka języka polskiego. Warszawa: Wydawnictwo Naukowe PWN. Peretti, Paula (1993): Die Rückfrage. Formen und Funktionen eines Sprechhandlungstyps im Deutschen und Spanischen anhand eines Corpus der gesprochenen Gegenwartssprache. München: Judicium Verlag.

Reis, Marga (1992): „Zur Grammatik und Pragmatik von Echo-w-Fragen.“ In: Rosengren, Inger (Hg.): Satz und Illokution. Bd. 1. Tübingen: Max Niemeyer Verlag. 213-261.

Rosengren, Inger (Hg.) (1987): Sprache und Pragmatik. Lunder Symposium 1986. Stockholm: Almqvist \& Wiksell.

Rosengren, Inger (Hg.) (1992): Satz und Illokution, Bd. 1. Tübingen: Max Niemeyer Verlag. 
Rost-Roth, Martina (2000): Nachfragen. Formen und Funktionen von Interrogationen im gesprochenen Deutsch. Freie Universität Berlin: Habilitationsschrift, Fachbereich Philosophie und Geisteswissenschaften.

Rost-Roth, Martina (2003): „Fragen - Nachfragen - Echofragen. Formen und Funktionen von Interrogationen im gesprochenen Deutsch.“ Linguistik-online 13 (1/2003), 325-378. (www.linguistik-online. de/13_01/rostRoth.html).

Rost-Roth, Martina (2011): „Form und Funktion von Interrogativen. Fragen in grammatischen Beschreibungen, empirischen Befunden und Lehrwerken für Deutsch als Fremdsprache." Linguistik-online 49 (5/2011), 91-117. (www.linguistik-online.de/49_11/rostRoth.html).

Schatte, Czesława/Schatte, Christoph (2005): „Rück- und Gegenfragen als reaktive Frageakte im deutsch-polnischen Vergleich.“ Scripta Neophilologica Posnaniensia VII, 97-110.

Selting, Margret (1987): Verständigungsprobleme. Eine empirische Analyse am Beispiel der Bürger-Verwaltungs-Kommunikation. Tübingen: Max Niemeyer Verlag.

Selting, Margret (1995): Prosodie im Gespräch. Aspekte einer interaktionalen Phonologie der Konversation. Tübingen: Max Niemeyer Verlag.

Wunderlich; Dieter (1986): „Echofragen.“ Studium Linguistik 20, 44-62.

Zifonun, Gisela/Hoffmann, Ludger/Strecker, Bruno (1997): Grammatik der deutschen Sprache. Berlin/ New York: Walter de Gruyter. 